\title{
On the Determination of Cauchy Surfaces from Intrinsic Properties ${ }^{\star}$
}

\author{
Robert Budic, James Isenberg ${ }^{\star}$, Lee Lindblom, and Philip B. Yasskin \\ Center for Theoretical Physics, Department of Physics and Astronomy, University of Maryland, \\ College Park, Maryland 20742, USA
}

\begin{abstract}
We consider the problem of determining from intrinsic properties whether or not a given spacelike surface is a Cauchy surface. We present three results relevant to this question. First, we derive necessary and sufficient conditions for a compact surface to be a Cauchy surface in a spacetime which admits one. Second, we show that for a non-compact surface it is impossible to determine whether or not it is a Cauchy surface without imposing some restriction on the entire spacetime. Third, we derive conditions for an asymptotically flat surface to be a Cauchy surface by imposing the global condition that it be imbedded in a weakly asymptotically simple and empty spacetime.
\end{abstract}

\section{Introduction}

In the initial value formulation of general relativity, one starts with initial data on a surface $S$ and evolves that data to produce a maximal 4-dimensional region, $D(S)$, the Cauchy development of $S$ (see [1] and pp. 244-255 of [2]). One goal of the initial value problem is to find sufficient intrinsic conditions on the surface $S$ and its data which will guarantee that its Cauchy development $D(S)$ is an inextendible spacetime. (The surfaces $A$ and $B$ in Figure 1 have developments which are extendible.) Such sufficient conditions are not known.

If $D(S)$ is extendible, then there always exists a maximal extension $M$ which is an inextendible spacetime. (This is easily proved using Zorn's lemma [3].) Then, one might ask whether there is some other surface $S^{\prime}$ whose development is all of $M$; i.e. $D\left(S^{\prime}\right)=M$. We prove a theorem which shows that if $S$ satisfies certain intrinsic conditions but is not a Cauchy surface for $M$, then $M$ has no Cauchy surface at all (i.e. $M$ is not globally hyperbolic). Stated another way, these intrinsic conditions on $S$ are sufficient to guarantee that if $M$ is globally hyperbolic then $S$ is

\footnotetext{
* This research was supported in part by the National Science Foundation grants PHY 70-022077 and PHY76-20029 as well as the National Aeronautics and Space Administration grant NGR 21-002-010

$\star \star$ National Science Foundation Pre-doctoral Fellow
} 

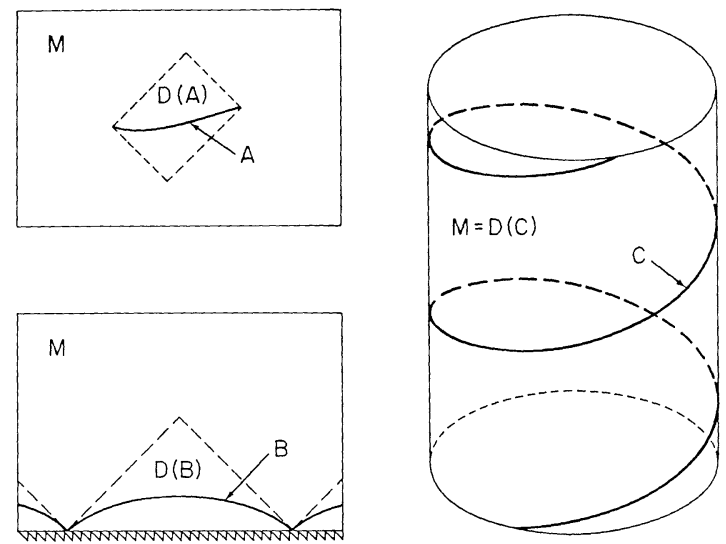

Fig. 1. Each of these diagrams represents a globally hyperbolic spacetime $M$ containing a connected spacelike submanifold $A, B$, or $C$ and its Cauchy development $D(A), D(B)$ or $D(C)$. Surface $A$ is an open ball. Surface $B$ intersects a singularity. Surface $C$ winds around a spacetime whose spatial topology is a 3-torus. None of the surfaces is a Cauchy surface for $M: D(A) \neq M, D(B) \neq M$, and $C$ is not acausal. None of the surfaces is compact: $A$ is open, $B$ runs off the manifold, and $C$ is unbounded

a Cauchy surface for $M$. Although motivated for an inextendible spacetime $M$ our theorem applies even if $M$ is extendible.

Most of the intrinsic conditions which we impose on $S$ are necessary requirements on any Cauchy surface: $S$ must be a connected, spacelike submanifold. However, as seen from the surfaces in Figure 1, these conditions are not sufficient to prove that $S$ is a Cauchy surface. If, we impose the additional intrinsic requirement that $S$ be compact, then our result goes through. In $\S$ II we prove,

Theorem 1. Let $S$ be a compact, connected, 3-dimensional manifold which is $C^{1}$ immersed as a spacelike submanifold of a spacetime $M$. If $M$ is globally hyperbolic then $S$ is a Cauchy surface.

It would be desirable to have a similar theorem in which the compactness assumption is replaced by some other conditions on $S$. However, without some additional conditions on the entire specetime $(M, g)$ such a theorem is impossible. This is shown by the following theorem (suggested by Robert Geroch) which is proved in $\S$ III.

Theorem 2. Let $S$ be a noncompact, 3-dimensional, spacelike imbedded submanifold of a time-oriented spacetime $(M, g)$. Then $S$ has a neighborhood $U$ which may be extended to a globally hyperbolic spacetime $\left(M^{\prime}, g^{\prime}\right)$ in which $S$ is not a Cauchy surface.

Theorem 2 indicates that for a noncompact surface $S$ in an arbitrary spacetime $M$, there are no conditions upon the intrinsic topology and geometry of $S$ which would be sufficient to guarantee that $S$ is a Cauchy surface. Even restrictions on the extrinsic geometry, describing the imbedding of $S$ in $M$, are not sufficient since the theorem shows that an entire neighborhood $U$ as well as $S$ is contained in some globally hyperbolic spacetime in which $S$ is not a Cauchy surface. 
In order to obtain a result in the noncompact case, similar to Theorem 1 , it is necessary to impose some condition on the entire spacetime such as requiring that Einstein's equations are satisfied everywhere in $M$. In $\S I V$ we present a result which requires the global condition that $M$ is weakly asymptotically simple and empty.

None of our proofs make any assumptions about the geometry or extrinsic curvature of $S$ in $M$. In fact our proofs only depend on the causal structure of the spacetime $M$ and not on any field equations or energy conditions.

The notation and terminology used in this paper are those of Hawking and Ellis [2]. Slight variations in some definitions exist in the literature. In particular, we use the term spacetime to refer to a connected 4-manifold $M$ having a Lorentz signature metric $g_{\alpha \beta}$. (Note that $M$ may be extendible.) We say that a surface $S$ is spacelike if every vector tangent to $S$ is spacelike, and we say that $S$ is acausal if every non-spacelike curve intersects $S$ at most once. Also, we define a Cauchy surface as an acausal surface $S$ such that $D(S)=M$. A slight modification of our proofs shows that if $S$ is only assumed to be non-timelike, then $S$ is an achronal surface such that $D(S)=M$ iff $M$ is globally hyperbolic. In $\S$ III there are two metrics on the same manifold $M^{\prime}$. To distinguish their causal structures, we will append the metric to the symbol as a subscript. For example, $J_{g}^{-}\left(C_{t}\right)$ is the past of the surface $C_{t}$ according to the metric $g$.

\section{Compact Surfaces}

To prove Theorem 1, we must show that every inextendible, non-spacelike curve intersects $S$ once and only once. We do this by first introducing two foliations of $M$ which Geroch [4] (see also [2], p. 212) has shown exist in every globally hyperbolic spacetime:

i) $M$ is foliated by a family of Cauchy surfaces $\left\{C_{t}, t \in \mathbb{R}\right\}$ which are the level surfaces of a time function $t: M \rightarrow \mathbb{R}$.

ii) $M$ is foliated by a congruence of timelike curves $\left\{\tau_{x}: x \in C_{0}\right\}$ where $\tau_{x} \cap C_{0}=\{x\}$. These curves define a $C^{1}$ projection $\pi: M \rightarrow C_{0}$ by mapping each point of $\tau_{x}$ into $x \in C_{0}$.

Using these foliations, we prove the theorem by successively showing that:

A) Every curve $\tau_{x}$ intersects $S$.

B) Every curve $\tau_{x}$ intersects $S$ at most once.

C) Every inextendible non-spacelike curve intersects $S$; i.e. $D(S)=M$.

D) Every non-spacelike curve intersects $S$ at most once; i.e. $S$ is acausal.

Hence, $S$ must be a Cauchy surface, which will complete the proof of the theorem.

\section{A) Every Curve $\tau_{x}$ Intersects $S$}

We must show that $\pi(S)=C_{0}$. Since $C_{0}$ is connected, it is sufficient to show that $\pi(S)$ is both open and closed within $C_{0}$.

First notice that as a compact immersed submanifold of $M, S$ is automatically imbedded; i.e. $S$ has the relative topology as a subset of $M$ (see [5], p. 22 and [6], p. 226). Hence, the restricted projection $\pi_{S}: S \rightarrow C_{0}$ is continuous. Since $S$ is compact, $\pi_{S}$ is a closed map (see [6], p. 226) and $\pi(S)$ is a closed subset of $C_{0}$. 
We now show that $\pi_{S}$ is a local diffeomorphism. For $p \in S$, the kernel of $\pi_{*}: T_{p}(M) \rightarrow T_{\pi(p)}\left(C_{0}\right)$ consists of the timelike vectors tangent to $\tau_{\pi(p)}$ at $p$. The kernel of the restriction $\pi_{S *}: T_{p}(S) \rightarrow T_{\pi(p)}\left(C_{0}\right)$ must be spacelike and so is trivial. Since $T_{p}(S)$ and $T_{\pi(p)}\left(C_{0}\right)$ are both 3-dimensional, $\pi_{S *}$ is an isomorphism. By the implicit function theorem (see [5], p. 24), $p$ has an open neighborhood $U C S$ such that the restriction $\pi_{U}: U \rightarrow \pi(U)$ is a diffeomorphism. Hence $\pi_{S}$ is a local diffeomorphism. Since a local homeomorphism is an open map, $\pi(S)$ is an open subset of $C_{0}$.

\section{B) Every Curve $\tau_{x}$ Intersects $S$ at Most Once}

Since $S$ is compact, for each $x \in C_{0}, \tau_{x} \cap S$ is compact and has a point $r_{x} \in \tau_{x} \cap S$ with maximum time; i.e. $t\left(r_{x}\right) \geqq t(q)$, for all $q \in \tau_{x} \cap S$. We must prove that $r_{x}$ is the only point in $\tau_{x} \cap S$. We do this by proving that the set $F=\left\{r_{x} \mid x \in C_{0}\right\}$ is all of $S$. Since $S$ is connected, it is sufficient to show that $F$ is both open and closed.

If $F$ were not open, there would exist a sequence of points $q_{n} \in S-F$ converging to a point $q \in F$. For each point $q_{n}$, there is a point $p_{n} \in F$ with $\pi\left(p_{n}\right)=\pi\left(q_{n}\right)$ and $t\left(p_{n}\right)>t\left(q_{n}\right)$. Since $S$ is compact, the sequence $p_{n}$ accumulates at some point $p \in S$. Since $\pi$ is continuous, $\pi(p)=\pi(q)$. Since $t$ is continuous, $t(p) \geqq t(q)$. Therefore $p=q \in F$. Now, every point $p \in S$ has an acausal neighborhood $U \subset S$. (This follows by a straightforward but tedious argument based on the strong causality assumption and the fact that $S$ is an imbedded spacelike submanifold.) Since the distinct sequences $p_{n}$ and $q_{n}$ both accumulate at $p$, there are points with timelike separation within $U$. This contradicts the acausality of $U$ and proves that $F$ is open.

To see that $F$ is closed, consider a sequence of points $p_{n} \in F$ converging to some point $q \in S$. Then there exists a point $p \in F$ with $\pi(p)=\pi(q)$. Since $F$ is open and $\pi_{S}$ is a local homeomorphism, $p$ has a neighborhood $U \subset F$ such that the restriction $\pi_{U}: U \rightarrow \pi(U)$ is a homeomorphism. Since $\pi$ is continuous the sequence $\pi\left(p_{n}\right)$ converges to $\pi(q)=\pi(p) \in \pi(U)$; and for large enough $n$ the sequence $\pi\left(p_{n}\right)$ remains in $\pi(U)$. Since $\pi_{U}^{-1} \circ \pi$ is continuous, the sequence $p_{n}=\pi_{U}^{-1} \circ \pi\left(p_{n}\right)$ converges to $\pi_{U}^{-1} \circ \pi(q)=\pi_{U}^{-1} \circ \pi(p)=p$ as well as to $q$. Hence $q=p \in F$ and $F$ is closed.

\section{C) Every Inextendible Non-Spacelike Curve Intersects $S$}

Since $S$ is compact, the time function $t$, restricted to $S$, assumes its maximum and minimum. Thus, $S$ lies between two surfaces $C_{t-}$ and $C_{t+}$ with $t^{-}<t(p)<t^{+}$for all $p \in S$. Any inextendible non-spacelike curve $\gamma$ must intersect both Cauchy surfaces $C_{t-}$ and $C_{t_{+}}$. Parametrize $\gamma$ so that $\gamma(0) \in C_{t-}$ and $\gamma(1) \in C_{t+}$; i.e. $t \circ \gamma(0)=t^{-}$and $t \circ \gamma(1)=t^{+}$. The projection of $\gamma$ into $S$ is the curve $\gamma_{S}=\pi_{S}^{-1} \circ \pi \circ \gamma$. Consider the continuous function $\Delta(\sigma)=t \circ \gamma(\sigma)-t \circ \gamma_{S}(\sigma)$. The function $\Delta$ measures the difference between the time of a point on $\gamma$ and the time of its projection in $S . \Delta(\sigma)$ is positive when $\gamma(\sigma)$ is in the future of $\gamma_{S}(\sigma)$, negative when $\gamma(\sigma)$ is in the past of $\gamma_{S}(\sigma)$, and zero when $\gamma(\sigma)=\gamma_{S}(\sigma)$. Since $\gamma_{S}$ lies in $S, t^{-}<t \circ \gamma_{S}(\sigma)<t^{+}$. Hence, $\Delta(0)=t^{-}-t \circ \gamma_{S}(0)<0$ while $\Delta(1)=t^{+}-t \circ \gamma_{S}(1)>0$. Since $\Delta$ is continuous, there exists $\sigma_{0} \in(0,1)$ such that $\Delta\left(\sigma_{0}\right)=0$. Therefore, $\gamma\left(\sigma_{0}\right)=\gamma_{S}\left(\sigma_{0}\right) \in S$ i.e. $\gamma$ intersects $S$ at the point $\gamma\left(\sigma_{0}\right)$.

\section{D) Every Non-Spacelike Curve Intersects $S$ at Most Once}

Let $\gamma$ be a future-directed, non-spacelike curve which intersects $S$ at $\gamma\left(\sigma_{0}\right)$. Let $\gamma_{S}=\pi_{S}^{-1} \circ \pi \circ \gamma$ be its projection into $S$ and consider the continuous function $\Delta(\sigma)=t \circ \gamma(\sigma)-t \circ \gamma_{S}(\sigma)$. Note that $\Delta\left(\sigma_{0}\right)=0$. 


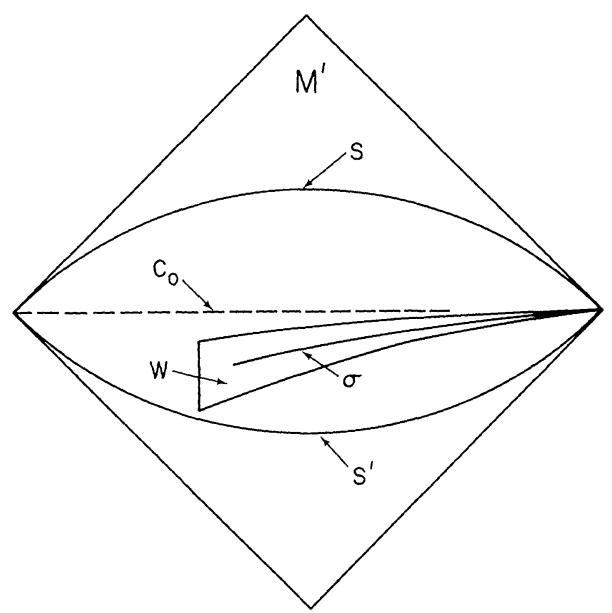

Fig. 2. This diagram illustrates how the two surfaces $S$ and $S^{\prime}$, the curve $\sigma$, and its open neighborhood $W$ are located within the spacetime region $M^{\prime}$. In the original metric $S$ and $S^{\prime}$ are Cauchy surfaces and $\sigma$ is noncausal. After modifying the metric in the region $W, \sigma$ becomes a timelike curve, the surface $S^{\prime}$ remains a Cauchy surface, but the surface $S$ is no longer a Cauchy surface

We now show that $\Delta$ is increasing in a neighborhood of $\sigma_{0}$. Let $U \subset S$ be an acausal neighborhood of $\gamma\left(\sigma_{0}\right)$. Notice that $\gamma\left(\sigma_{0}\right) \in \operatorname{int} D(U)$. Hence there exists a $\delta>0$ such that $\gamma(\sigma) \in \operatorname{int} D(U)$ for all $\sigma \in\left(\sigma_{0}-\delta, \sigma_{0}+\delta\right)$. If $\gamma(\sigma) \in D^{+}(U)$ then the curve $\tau_{\pi(\gamma(\sigma))}$ must intersect $U$ at $\gamma_{S}(\sigma)$ in the past of $\gamma(\sigma)$, i.e. $\Delta(\sigma)>0$. Similarly if $\gamma(\sigma) \in D^{-}(U)$ then $\Delta(\sigma)<0$. If $\sigma \in\left(\sigma_{0}-\delta, \sigma_{0}\right)$ and $\gamma(\sigma) \in D^{+}(U)$ then the curve $\tau_{\pi(\gamma(\sigma))}$ from $\gamma_{S}(\sigma) \in U$ to $\gamma(\sigma)$ followed by the curve $\gamma$ from $\gamma(\sigma)$ to $\gamma\left(\sigma_{0}\right) \in U$ would be a future-directed, non-spacelike curve which intersects $U$ twice. This is impossible because $U$ is acausal. Therefore, if $\sigma \in\left(\sigma_{0}-\delta, \sigma_{0}\right)$ then $\gamma(\sigma) \in D^{-}(U)$ and $\Delta(\sigma)<0$. Similarly, if $\sigma \in\left(\sigma_{0}, \sigma_{0}+\delta\right)$ then $\Delta(\sigma)>0$. Since $\Delta$ is continuous, it must be increasing in some neighborhood of $\sigma_{0}$.

Therefore, $\Delta$ is a continuous function which is increasing whenever it is zero. Thus, $\Delta$ can have at most one zero and $\gamma$ can intersect $S$ at most once.

\section{Noncompact Surfaces}

Our proof of Theorem 2 is constructive. Out of the given spacetime $(M, g)$ containing $S$, we construct the neighborhood $U$ containing $S$ and the spacetime extension of $U$, called $\left(M^{\prime}, g^{\prime}\right)$. We choose the sets $M^{\prime}$ and $U \subset M^{\prime}$ as subsets of $M$. Then we construct the new metric $g^{\prime}$ by modifying the metric $g$ within a set $W$ which is in the past of $S$ and outside of $U$. The modification consists of tipping the light cones so that a certain curve $\sigma$ contained entirely within $W$ (and therefore in the past of $S$ ) becomes a future inextendible timelike curve according to $g^{\prime}$. The existence of the curve $\sigma$ in the past of $S$ shows that $S$ is not a Cauchy surface for $\left(M^{\prime}, g^{\prime}\right)$. However, we finally show that there is a surface $S^{\prime}$ in the past of $W$ which remains a Cauchy surface after the metric is modified, so that $\left(M^{\prime}, g^{\prime}\right)$ is globally hyperbolic. Figure 2 shows where the curve $\sigma$ and the neighborhood $W$ are located 


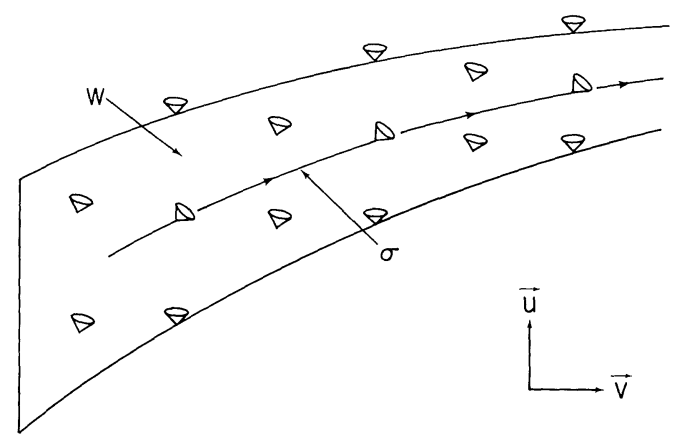

Fig. 3. This diagram illustrates how the metric is modified in the region $W$. In the metric $g$ the light cones are at $45^{\circ}$ in this diagram and the curve $\sigma$ is noncausal. The light cones shown in the figure are those of the modified metric $g^{\prime}$. Note that in the modified metric $\sigma$ is a future directed timelike curve which never intersects $S$

relative to the surfaces $S$ and $S^{\prime}$ within $M^{\prime}$. Figure 3 shows how the metric is modified within $W$ by tipping the light cones.

Proof of Theorem 2. The manifold $M^{\prime} C M$ is chosen so that $S$ is a Cauchy surface for $\left(M^{\prime}, g\right)$. To do this, we first find a neighborhood $N \subset M$ in which $S$ is achronal. (The existence of such a neighborhood is guaranteed by Lemma 2 in the Appendix.) Then we take $M^{\prime}=D_{g}(S, N)$.

Since $\left(M^{\prime}, g\right)$ is globally hyperbolic, it can be foliated by surfaces $C_{t}$ and by curves $\tau_{x}$ as described in $\S$ II. Like $S$, all of the surfaces $C_{t}$ are noncompact. Also, as in $\S I I$, the projection along the curves $\tau_{x}$ is denoted by $\pi$. For the present proof, we choose the curves $\tau_{x}$ to be everywhere orthogonal to the surfaces $C_{t}$ and we choose the surfaces $C_{t}$ so that $S=C_{T}$ for some $T>0$. The open set $U=\left\{p \in M^{\prime}: t(p)>0\right\} \subset M^{\prime} \subset M$ is the neighborhood of $S$ required by the theorem. The set $M^{\prime}$ is the manifold of the extension $\left(M^{\prime}, g^{\prime}\right)$.

Our construction of the curve $\sigma$ and the set $W$ (within which the metric is to be modified) begins with the specification of a curve $\sigma_{0}$ and a set $\Sigma$, both contained in the noncompact surface $C_{0}$. We let $\sigma_{0}:[0, \infty) \rightarrow C_{0}$ be any smooth imbedded spacelike curve which is not confined to any compact subset of $C_{0}$. Hence, $\sigma_{0}$ has no endpoint as its parameter $z$ approaches infinity. We let $\Sigma \subset C_{0}$ be a neighborhood of the curve $\sigma_{0}$ in which each point $q \in \Sigma$ lies on a unique geodesic (within $\Sigma$ ) which is orthogonal to $\sigma_{0}$. (The existence of such a neighborhood is guaranteed by Lemma 1 in the Appendix.) We demand that $\Sigma$ be chosen small enough so that there is an upper bound to the proper distance from any point in $\Sigma$ to the curve $\sigma_{0}$ along an orthogonal geodesic.

We now extend the parameter $z$ along $\sigma_{0}$ to a function $z$ on $\Sigma$ by demanding that $z$ be constant along the geodesics in $\Sigma$ which are orthogonal to $\sigma_{0}$. Furthermore, we extend the function $z$ to the region $\pi^{-1}(\Sigma)=\{q: \pi(q) \in \Sigma\}$ by letting $z(q)=z(\pi(q))$.

We now complete the construction of the curve $\sigma$ and the set $W$. For $z \geqq 1$, let $\sigma(z)=\tau_{\sigma_{0}(z)} \cap C_{t^{\prime}(z)}$ where $t^{\prime}(z)=-\frac{1}{2} T \exp (-z)$. Also, let

$$
W=\left\{q \in \pi^{-1}(\Sigma):-\frac{3}{4} T \exp \left(-z[q], \leqq t(q) \leqq-\frac{1}{4} T \exp (-z[q])\right\} .\right.
$$


This somewhat peculiar definition of $W$ is chosen to ensure that for all $t<0$, the set $J_{g}^{-}\left(C_{t}\right) \cap W$ has compact closure. (Recall that on this set, the function $z$ is bounded.)

The metric is modified on the set $W$ by tilting the cones of $g$ so that $\sigma$ becomes a timelike curve. To specify the tilting, we use three vector fields:

$$
u^{\alpha}=-\left(-g^{\mu v} \partial_{\mu} t \partial_{v} t\right)^{-1 / 2} g^{\alpha \beta} \partial_{\beta} t, \quad v^{\alpha}=\left(g^{\mu v} \partial_{\mu} z \partial_{v} z\right)^{-1 / 2} g^{\alpha \beta} \partial_{\beta} z,
$$

and $k^{\alpha}$, which is the tangent to the curve $\sigma$. Note that $u^{\alpha} v^{\beta} g_{\alpha \beta}=0$, because $u^{\alpha}$ is tangent to the curves $\tau_{x}$ while $z$ is constant on these curves. Also note that $k^{\alpha} u^{\beta} g_{\alpha \beta}<0$ and $k^{\alpha} v^{\beta} g_{\alpha \beta}>0$, since $t$ and $z$ increase along $\sigma$. We define the function $\phi$ along $\sigma$ as the angle between $k^{\alpha}$ and $u^{\alpha}$ in the positive definite metric $O_{\alpha \beta}=g_{\alpha \beta}+2 g_{\alpha \mu} u^{\mu} g_{\beta v} u^{\nu}$. Finding that $\cos \phi$ and $\sin \phi$ are both positive definite, we see that $0<\phi<\frac{\pi}{2}$. We extend the definition of the function $\phi$ to all of $W$ by demanding $\phi(p)=\phi(q)$ iff $z(p)=z(q)$. We also need a smooth function $f: M^{\prime} \rightarrow[0,1]$, which vanishes everywhere in $M^{\prime}-W$ and assumes the value $f=1$ on $\sigma$. The existence of this function is demonstrated in [5], p. 11. The modified metric $g^{\prime}$ is now defined by tilting the light cones of $g$ by the amount $\theta=\frac{1}{2} \phi f:$ let $l^{\alpha}=-u^{\alpha} \cos \theta$ $+v^{\alpha} \sin \theta$; then $g_{\alpha \beta}^{\prime}=O_{\alpha \beta}-2 O_{\alpha \mu} l^{\mu} O_{\beta v} l^{v}$.

We have now constructed the spacetime $\left(M^{\prime}, g^{\prime}\right)$ as an extension of the neighborhood $U$ containing $S$ (inside $U, g^{\prime}=g$ ). To complete the proof of the theorem, we must show that $\left(M^{\prime}, g^{\prime}\right)$ is globally hyperbolic and that $S$ is not a Cauchy surface for $\left(M^{\prime}, g^{\prime}\right)$.

We show that $\left(M^{\prime}, g^{\prime}\right)$ is globally hyperbolic by showing that $S^{\prime}=C_{-T}$ is a Cauchy surface; i.e. that any inextendible causal curve $\gamma$ must intersect $S^{\prime}$ once and only once. Consider such a curve. If $\gamma$ does not intersect the region $W$, it follows that $\gamma$ is a timelike curve in the original metric $g$. Since $S^{\prime}$ is a Cauchy surface for $\left(M^{\prime}, g\right), \gamma$ must intersect $S^{\prime}$ exactly once.

Now assume that $\gamma$ intersects the region $W$ at some point $p$. Then as $\gamma$ proceeds toward the past from the point $p$, it can only intersect $W$ within $J_{g^{\prime}}^{-}\left(C_{t(p)}\right) \cap W$. However, since the $\tau_{x}$ congruence remains timelike in the new metric $\left(g_{\alpha \beta}^{\prime} u^{\alpha} u^{\beta}<0\right)$, we have that $J_{g^{\prime}}^{-}\left(C_{t(p)}\right)=J_{g}^{-}\left(C_{t(p)}\right)$. So $\gamma$ can only intersect $W$ within $J_{g}^{-}\left(C_{t(p)}\right) \cap W$. We saw earlier that this set is compact. Further, $t$ is a time function for $\left(M^{\prime}, g^{\prime}\right)$ since $\left(g^{\prime-1}\right)^{\alpha \beta} \partial_{\alpha} t \partial_{\beta} t=g^{\alpha \beta} \partial_{\alpha} t \partial_{\beta} t \cos 2 \theta<0$. Consequently $\left(M^{\prime}, g^{\prime}\right)$ is strongly causal and cannot have any inextendible causal curves partially imprisoned in any compact set (Proposition 6.4.7 of [2]). Hence $\gamma$ must eventually leave and remain outside of $W$. Afterward, the metric along $\gamma$ will be identical to $g$. Consequently, $\gamma$ must intersect $S^{\prime}$. This shows that $D_{g^{\prime}}\left(S^{\prime}\right)=M^{\prime}$. The metric $g^{\prime}$ differs from $g$ only in the region $W$, which lies entirely in the future of $S^{\prime}$. Consequently the entire past of $S^{\prime}$ is identical in both metrics. As a result no non-spacelike curve $\gamma$ (according to $g^{\prime}$ ) can intersect $S^{\prime}$ more than once, since no non-spacelike curve (according to $g$ ) intersects $S^{\prime}$ more than once. Thus $S^{\prime}$ is achronal, and $\left(M^{\prime}, g^{\prime}\right)$ must be globally hyperbolic.

The last step in the proof is to argue that the surface $S$ is not a Cauchy surface for $\left(M^{\prime}, g^{\prime}\right)$. We accomplish this by showing that the curve $\sigma$ is a future inextendible timelike curve which does not intersect $S$. The curve $\sigma$ was specifically constructed so that it does not intersect $S$. The metric $g^{\prime}$ was specifically designed so that $\sigma$ is future timelike, since $g_{\alpha \beta}^{\prime} k^{\alpha} k^{\beta}=-O_{\alpha \beta} k^{\alpha} k^{\beta} \cos 2(\phi-\theta)<0$. If the curve $\sigma$ were not 
inextendible, there would be a point $p$, such that $\sigma$ would enter and remain within every open neighborhood of $p$. It would also follow that $\pi(p)$ would be an endpoint of the curve $\sigma_{0}=\pi \circ \sigma$. But $\sigma_{0}$ was specifically chosen to have no endpoint; hence $\sigma$ must also be inextendible. Thus $S$ cannot be a Cauchy surface for $\left(M^{\prime}, g^{\prime}\right)$.

\section{Asymptotically Flat Surfaces}

As we have seen from Theorem 2, it is not possible to determine whether or not a non-compact surface is a Cauchy surface simply by imposing intrinsic conditions. One possible global condition would be to require that Einstein's equations (with suitable energy conditions) be satisfied at every point. Then, one might conjecture: If $M$ is a globally hyperbolic spacetime satisfying Einstein's equations and if $S$ is a spacelike surface which is in some sense spatially asymptotically flat [7-9], with one asymptotic region, then $S$ is a Cauchy surface for $M$.

We cannot prove this conjecture, but we can prove a closely related result. Instead of imposing Einstein's equations at each point as our global assumption, we require that the spacetime, $M$, be weakly asymptotically simple and empty. Also, we require that the surface $S$ approaches spatial infinity (i.e. for every neighborhood $U$ of spatial infinity, $i_{0}$, the set $S-U$ is compact).

Our preliminary result is: Let $S$ be a connected, 3-dimensional manifold which is $C^{1}$ imbedded as a spacelike submanifold of a spacetime $M$. If $M$ is globally hyperbolic and weakly asymptotically simple and empty with one asymptotic region and if $S$ approaches spatial infinity, then $S$ is a Cauchy surface.

The proof of our preliminary result is nearly identical to that given in $\S$ II for Theorem 1. Hence we omit the details here, except to point out the one major modification. In general, a non-compact surface cannot be bounded between two leaves of a given Cauchy foliation, even if the surface is required to be compact outside every neighborhood of spatial infinity. We therefore use a different foliation (see [2], pp. 221 and 313). Its leaves are partial Cauchy surfaces $C_{t}$ which become Cauchy surfaces when adjoined to a piece of $\mathscr{I}$. For each $t$, the region $U_{t}$ between $C_{t}$ and $C_{-t}$ is a neighborhood of $i_{0}$. Since $S-U_{t}$ is compact, $S$ may be bounded between two leaves of this new foliation.

\section{Appendix}

Let $S$ be a submanifold of a manifold $M$ with metric $g$. Assume $\operatorname{dim} S<\operatorname{dim} M$. An $S$-normal neighborhood is defined as an open set $N \subset M$ such that every point $q \in N$ is connected to $S \cap N$ by a unique geodesic which is contained within $N$, and which is normal to $S$. If $Q \subset S \cap N$, then $N$ is called an $S$-normal neighborhood of $Q$. In particular, the usual definition of a normal neighborhood of a point is just a $p$-normal neighborhood of the point $p$.

Lemma 1. If $\sigma$ is an imbedded submanifold of a manifold $C$ with positive definite metric $g$, then $\sigma$ has an $\sigma$-normal neighborhood.

Lemma 1 is a slight modification of a theorem presented by Milnor and Stasheff [10], and its proof is easily modeled after their proof.

In the case of a manifold with an indefinite metric, we prove a similar lemma: 
Lemma 2. If $S$ is a spacelike, 3-dimensional imbedded submanifold of a timeoriented spacetime $M$, then $S$ has an $S$-normal neighborhood in which it is achronal.

Proof. We first note that every neighborhood of $p \in S$ contains an $S$-normal neighborhood of $p$, since the exponential map is well-defined on a small enough neighborhood.

For each $p \in S$, let $U_{p}$ be an $S$-normal neighborhood of $p$. Then $M_{1}=\bigcup_{p \in S} U_{p}$ is an open neighborhood of $S$ within $M$. Since $M_{1}$ is paracompact, it has an open cover $V=\left\{V_{i}\right\}$ such that for each point $q \in M_{1}$ there is another point $r(q) \in S$ such that $\bigcup\left\{V_{i} \in V: q \in V_{i}\right\} \subset U_{t}(q)$. (See [6], pp. 167 and 168.) Now each $p \in S$ belongs to some $V_{i} \in V$. Let $W_{p}$ be a new surface normal neighborhood of $p$ contained in some $V_{i}$. Then $M_{2}=\bigcup_{p \in S} W_{p}$ is a new open neighborhood of $S$ within $M$.

We claim that $M_{2}$ is an $S$-normal neighborhood of $S$. To see this, let $q \in M_{2}$. For each $W_{p}$ containing $q$ there is a unique normal geodesic within $W_{p}$ connecting $q$ to $S$. However, $q$ may belong to many $W_{p}$. We need to prove that all the normal geodesics coincide. But since each $W_{p}$ is contained in some $V_{i}$, we have

$$
\bigcup\left\{W_{p}: q \in W_{p}\right\} \subset \bigcup\left\{V_{i}: q \in V_{i}\right\} \subset U_{r}(q) \text {. }
$$

Thus the normal geodesic within each $W_{p}$ must coincide with the unique normal geodesic within $U_{r}(q)$ and thus they must all coincide within $M$.

To see that $S$ is achronal within $M_{2}$, notice that $M_{2}$ has a time function which measures proper time along the normal geodesics and is zero on $S$. Since $S$ is the level surface of a time function on $M_{2}$, it is achronal within $M_{2}$.

\section{References}

1. Choquet-Bruhat, Y., Geroch, R.: Commun. math. Phys. 14, 329-335 (1969)

2. Hawking, S.W., Ellis, G.F.R.: Large scale structure of space-time. Cambridge: University Press 1973

3. Kelley,J.L.: General topology, p. 33. Toronto-New York-London: Van Nostrand 1955

4. Geroch, R. : J. Math. Phys. 11, 437-449 (1970)

5. Warner,F.W.: Foundations of differentiable manifolds and Lie groups. Glenview, Ill.: Scott, Foresman 1971

6. Dugundji,J.: Topology. Boston: Allyn and Bacon 1966

7. Geroch, R. : J. Math. Phys. 13, 956-968 (1972)

8. Gannon,D.: J. Math. Phys. 16, 2364-2367 (1975)

9. Misner,C.W.: Waves, newtonian fields and coordinate functions. In : Proceedings on the theory of gravitation, pp. 189-205. Paris: Gauthier-Villars 1964

10. Milnor,J.W., Stasheff,J.D. : Characteristic classes, p. 115. Princeton: University Press 1974

Communicated by R. Geroch

Received October 3, 1977; in revised form February 17, 1978 
